Journal of

Applied

Crystallography

ISSN 0021-8898

Received 29 August 2002

Accepted 14 April 2003
C) 2003 International Union of Crystallography Printed in Great Britain - all rights reserved

\section{Co-crystallization of poly(ethylene-butene) copolymers and paraffin molecules in decane studied with small-angle neutron scattering ${ }^{1}$}

\author{
A. Radulescu, ${ }^{a} *$ D. Schwahn, ${ }^{a}$ D. Richter ${ }^{\mathrm{a}}$ and L .J. Fetters ${ }^{\mathrm{b}}$ \\ anstitute for Solid State Research, Research Center Jülich GmbH, D52425 Jülich, Germany, and \\ ${ }^{\mathbf{b}}$ School of Chemical Engineering, Cornell University, Ithaca, NY 14853, USA. Correspondence e- \\ mail: a.radulescu@fz-juelich.de
}

\begin{abstract}
PEB- $n$ semicrystalline poly(ethylene-butene) random copolymers may become important in industrial applications because of their capability to modify the paraffin crystals and to improve the viscoelastic properties of crude oil and refined fuel. The copolymer-paraffin interaction is rather complex and is not yet understood. Therefore, these polymers may also be of much interest in basic research. Systematic small-angle neutron scattering investigations of different polymer-paraffin systems have shown that PEB- $n$ copolymers are selective in their paraffin-modification capacity, depending on the crystalline degree which is tuned by the number ' $n$ ' of ethyl side branches/100 backbone $\mathrm{C}$ atoms. Two mechanisms of polymer-paraffin interaction have been identified: on the one hand, co-crystallization of the paraffin and copolymer within thin platelets occurs as a consequence of a good fit between the self-assembling properties of the investigated polymer/paraffin pairs (PEB-7.5/ $\mathrm{C}_{36}$ and $\mathrm{PEB}-11 / \mathrm{C}_{24}$ ); on the other hand, primordial polymer aggregates act as a template for a subsequent paraffin crystallization when the polymer has a higher self-assembling point (PEB-7.5/ $\mathrm{C}_{24}$ ) than the paraffin. Both mechanisms were found for the PEB-7.5/ $\mathrm{C}_{30}$ mixture.
\end{abstract}

\section{Introduction}

Under wintry conditions, middle distillate fuel can clog filters by precipitating large paraffin crystals and thereby prevent engine operation. Polymeric additives can interact favorably with paraffin (long-chain $\mathrm{C}_{n} \mathrm{H}_{2 n+2}$ alkanes or simply $\mathrm{C}_{n}$ ) and modify the crystal morphology to smaller units. The mechanism of polymer and paraffin interaction is still not understood. In dilute solutions of decane, diblock copolymers of polyethylene and poly(ethylene-propylene) (PE-PEP) or poly(ethylene-butene) (PE-PEB) spontaneously assemble to form hairy platelets (Richter et al., 1997; Lin \& Gast, 1997), the perpendicular dimensions of which are controlled by the molecular weight and the ratio of the two block lengths. This process is driven by the crystallization of the PE blocks, forming a core which is surrounded by amorphous PEP or PEB brushes. This structure provides a large surface area for the nucleation of paraffin (Leube et al., 2000; Monkenbusch et al., 2000). These observations stimulated further exploration of other polymer architectures representing a combination of semicrystalline and amorphous segments in an alternating manner. Virtually, random copolymers of ethylene and butene (designated as PEB- $n$ with ' $n$ ' the number of ethyl side branches/100 backbone carbons) are promising candidates.

\footnotetext{
$\mathbf{1}_{\mathbf{1}}$ This paper was presented at the XIIth International Conference on SmallAngle Scattering, Venice, Italy, 25-29 August 2002.
}

Small-angle neutron scattering (SANS) experiments have shown that in solution these copolymers form needle-shape objects in the low-temperature range and that the transition temperature becomes lower with increasing $n$ (Schwahn et al., 2002a; Ashbaugh et al., 2002). Depending on the graded ethylene content of the backbone, i.e. on $n$ and therefore on the crystalline degree, the needles are either rather open, as in the case of the PEB-11 copolymer, or built up by alternating amorphous-crystalline sequences, as in the case of the higher crystalline PEB-7.5 copolymer.

Structures of different PEB- $n$ polymer-paraffin systems obtained from systematic SANS investigation were previously summarized in terms of 'structural diagrams' represented in temperature versus wax volume-fraction maps (Ashbaugh et $a l ., 2002)$. It was shown that the modification of the paraffin crystal is strongly determined by the crystalline degree of the PEB- $n$ copolymers. A high polymer efficiency was observed in the case of a good fit between the self-assembling temperatures of the investigated polymer/paraffin pairs (PEB-7.5/ $\mathrm{C}_{36}$ and PEB-11/ $\mathrm{C}_{24}$ ) and the efficiency became markedly reduced when the polymer had a higher self-assembling temperature than the paraffin (PEB-7.5/ $\mathrm{C}_{24}$ ).

In this paper we will present the aggregation behavior of the PEB-7.5 copolymer alone and in combination with the $\mathrm{C}_{36}, \mathrm{C}_{30}$ and $\mathrm{C}_{24}$ paraffins in decane. We applied the SANS contrastvariation technique in order to specify the conformation of the 
copolymer and paraffin separately. The aggregated structures will be identified by a semi-quantitative interpretation of the data in terms of power laws. The common aggregation mechanisms will be discussed for each of the paraffin/polymer combinations in parallel with the analysis of the precipitation temperature of both materials in decane.

\section{Theory}

The scattering cross section $\mathrm{d} \Sigma / \mathrm{d} \Omega(Q)$ of a multi-component system can be described in terms of partial structure factors $S_{i j}(Q)$; for a ternary system $\mathrm{d} \Sigma / \mathrm{d} \Omega(Q)$ is represented by

$$
\begin{aligned}
\mathrm{d} \Sigma / \mathrm{d} \Omega(Q)= & \left(\rho_{p}-\rho_{s}\right)^{2} S_{p p}(Q)+2\left(\rho_{p}-\rho_{s}\right)\left(\rho_{w}-\rho_{s}\right) S_{p w}(Q) \\
& +\left(\rho_{w}-\rho_{s}\right)^{2} S_{w w}(Q),
\end{aligned}
$$

where $Q$ is the modulus of the scattering vector, $4 \pi \sin \theta / \lambda$ with $\theta$ being half the scattering angle and $\lambda$ being the wavelength, subscripts $p$ and $w$ indicate the polymer and paraffin (wax), respectively, and the solvent $s$ was chosen as the reference component. The factor $\Delta \rho_{i j}=\left(\rho_{i}-\rho_{j}\right)$ represents the difference between the coherent scattering length densities of the components $i$ and $j$ and is proportional to the scattering contrast. The scattering-length density $\rho$ of all three components can be changed within given limits by hydrogenation or deuteration. This allows preparation of samples with either the polymer $\left[S_{p p}(Q)\left(\rho_{w}-\rho_{s}=0\right)\right]$ or the paraffin $\left[S_{w w}(Q)\left(\rho_{p}-\right.\right.$ $\left.\left.\rho_{s}=0\right)\right]$ 'visible'.

At high temperatures, the polymer and paraffin molecules are dissolved and provide scattering from the single units. At low temperature, large objects are formed by crystallization. The scattering from those objects can often be characterized by power laws according to

$$
\mathrm{d} \Sigma / \mathrm{d} \Omega(Q) \simeq Q^{-\alpha}
$$

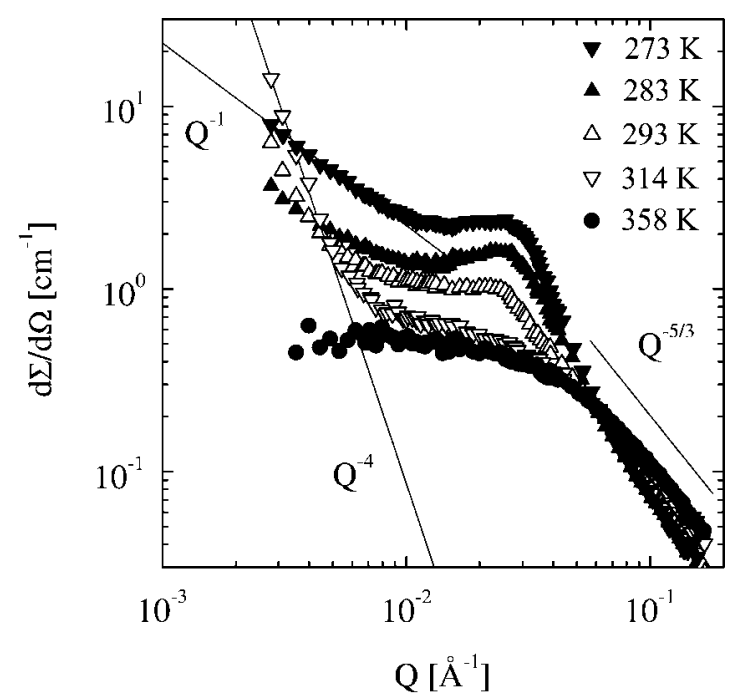

Figure 1

Small-angle scattering cross section from a solution of PEB-7.5 random copolymers in d-22 $\left(\Phi_{\mathrm{pol}}=1 \%\right)$. The solid lines indicate the asymptotic power law scattering behavior according to equation (2). with the exponent $\alpha$ being characteristic for certain spatial arrangements (Higgins \& Benoit, 1994). For single polymers, one finds $\alpha=1 / v$ (Flory exponent $v=3 / 5$ ) at large $Q$ for swollen chains in a good solvent, and $\alpha=2$ for chains in $\theta$ solvent condition. For aggregated structures, one finds $\alpha=1$ for rods and $\alpha=2$ for platelets. Aggregates representing large three-dimensional objects $(R>1 / Q)$ with sharp interfaces give rise to Porod scattering with $\alpha=4$. Exponents of $\alpha<3$ and $3<$ $\alpha<4$ can represent mass or surface fractal structures, while $\alpha>$ 4 may be characteristic for diffuse interfaces.

\section{Experiment}

Random copolymers of ethylene and butene of well defined molecular weight and composition were prepared by anionic polymerization of butadiene with random alternation of 1,4and 1,2-monomers (Morton \& Fetters, 1975). Hydrogenation then lead to ethyl side branches of an otherwise linear PE, denoted PEB- $n$. The ethyl-side-branch content was checked by nuclear magnetic resonance. The copolymer PEB- $n$ with $n=7.5$ had a molecular weight of about $6 \mathrm{~kg} \mathrm{~mol}^{-1}$.

The SANS experiments were performed at the FRJ-2 research reactor in Jülich in a $Q$ range of $0.002<Q<0.14 \AA^{-1}$. The data were corrected for scattering from an empty cell and calibrated in absolute units by a Lupolen secondary standard (Schwahn et al., 1990). The self-assembling behavior of PEB7.5 in decane was established by investigating a $1 \%$ polymer solution over a large temperature range, between the singlecoil and the aggregate regime (from 358 to $253 \mathrm{~K}$ ). Several solutions of PEB-7.5 copolymer and $\mathrm{C}_{24}, \mathrm{C}_{30}$ or $\mathrm{C}_{36}$ paraffins were then explored. Two paraffin volume fractions $\left(\Phi_{\text {wax }}=0.5\right.$ and $4 \%$ ) were mixed with $0.6 \%$ polymer for each of the paraffins studied. The solutions were first heated to $358 \mathrm{~K}$ to measure the polymer and paraffin single-chain conformation and were then gradually cooled to explore the aggregation behavior. The samples with $\mathrm{C}_{24}, \mathrm{C}_{30}$ and $\mathrm{C}_{36}$ were investigated down to 253,263 and $273 \mathrm{~K}$, respectively. In order to achieve maximum contrast and to minimize the incoherent background, the solutions were always prepared in deuterated decane (d-22). The contrast conditions were chosen according to equation (1) in order to study separately (i) the polymer conformation and (ii) the paraffin conformation within the aggregates, e.g. (i) fully protonated polymers and matched paraffin molecules $\left(\rho_{w}=\rho_{s}\right)$, and (ii) protonated paraffin and matched polymers $\left(\rho_{p}=\rho_{s}\right)$.

\section{Results and discussion}

Fig. 1 displays the scattering profiles of a 1\% PEB-7.5 copolymer solution in d-22 measured at temperatures between 358 and $253 \mathrm{~K}$. Self-assembly starts at a rather high temperature, above $313 \mathrm{~K}$, and shows a multiple evolution: (i) the formation of large compact aggregates at $314 \mathrm{~K}$, as proven by the Porodlike scattering at low $Q$, which coarsen or decrease in size at decreasing temperature; (ii) the evolution of a correlation peak in the intermediate $Q$ range; and (iii) the development of needle-like aggregates, as proven by the $Q^{-1}$ power law at 
$283 \mathrm{~K}$ and below. The peak denotes a correlation length of about $250 \AA$, which possibly signifies a periodic structure along the one-dimensional objects. At large $Q$, a $Q^{-1 / v}$ behavior with the Flory exponent $v=0.6$ is observed. Their intensity level decreases at decreasing temperature as the polymer single coils aggregate.

Fig. 2 represents scattering profiles obtained under polymer (full dots) and paraffin (open dots) contrast conditions from two PEB-7.5/ $\mathrm{C}_{36}$ solutions. In both solutions the polymer had a concentration of $\Phi_{\mathrm{pol}}=0.6 \%$, while in Figs. $2(a)$ and $2(b)$ the scattering patterns of the solutions with $\Phi_{\mathrm{wax}}=0.5 \%$ at $293 \mathrm{~K}$ and $\Phi_{\text {wax }}=4 \%$ at $313 \mathrm{~K}$ are shown. At lower paraffin concentration, the polymer and paraffin co-crystallize into two-dimensional structures. The polymer profile shows an oscillatory behavior: a minimum at around $Q=0.035 \AA^{-1}$ is followed by a peak at around $Q=0.06 \AA^{-1}$. Such a profile is indicative of a polymer brush leaking out on both sides of a platelet-like core (Richter et al., 1997). For the paraffin profile, a $Q^{-2}$ behavior is extended over a wide $Q$ range. Applying the two-dimensional Guinier approximation (Schwahn et al., $2002 b$ ) the deviation from the power law at high $Q$ yields a platelet thickness of about $55 \AA$. Driven by the paraffin tendency to form platelets at high temperatures (Ashbaugh et al., 2002), the interaction of hexatriacontane with PEB-7.5 thus yields a core structure formed by the paraffin and the crystallizing parts of the copolymer surrounded by brushes of

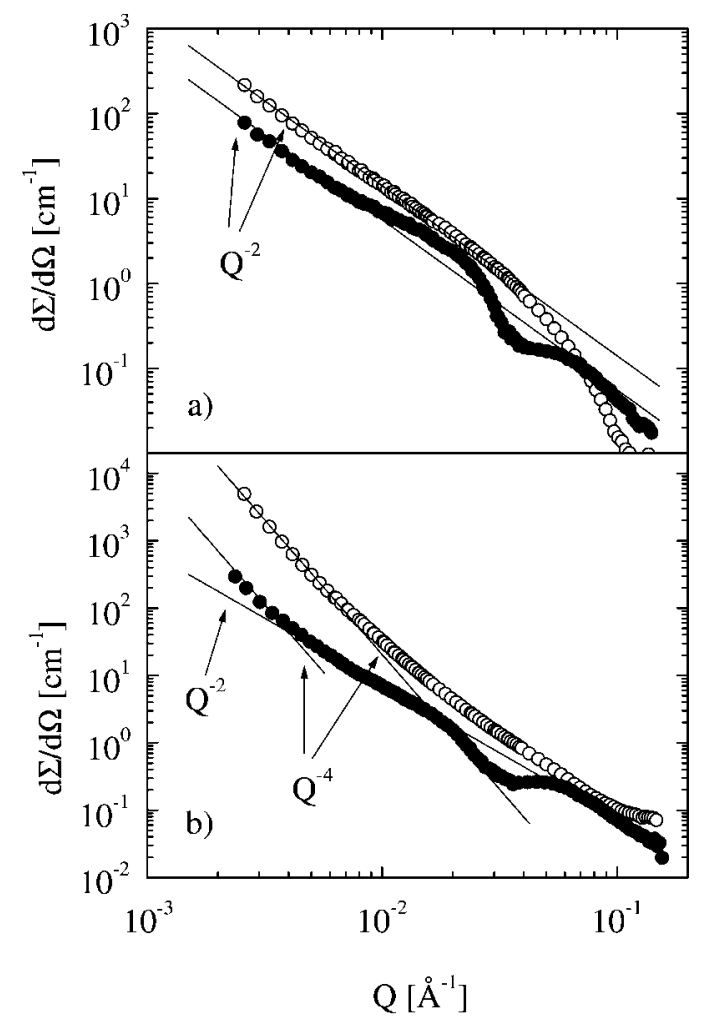

Figure 2

Small-angle scattering cross sections obtained from mixed solutions of PEB-7.5 $\left(\Phi_{\mathrm{pol}}=0.6 \%\right)$ and $\mathrm{C}_{36}$ in d-22 for $(a) \Phi_{\mathrm{wax}}=0.5 \%$ at $293 \mathrm{~K}$ and (b) $\Phi_{\text {wax }}=4 \%$ at $313 \mathrm{~K}$. The solid lines indicate the asymptotic power law scattering behavior at low $Q$. Symbols denote either the polymer (filled circles) or paraffin (open circles) contrast conditions. the swollen amorphous polymer segments. For the higher paraffin concentration in Fig. 2(b), the same principle structure is formed but additional large three-dimensional objects, mainly composed of paraffin, are nucleated and grow on the platelet surface.

Fig. 3(a) displays the scattering cross sections of a PEB-7.5/ $\mathrm{C}_{30}$ solution $\left(\Phi_{\mathrm{pol}}=0.6 \%\right.$ and $\left.\Phi_{\mathrm{wax}}=4 \%\right)$ measured at $293 \mathrm{~K}$ under the polymer and paraffin contrast conditions. The scattering patterns look very similar to those obtained from the high-paraffin-concentration $\mathrm{PEB}-7.5 / \mathrm{C}_{36}$ solution. This proves again the co-crystallization of polymer and paraffin in hairy platelets followed by a subsequent nucleation of large paraffin aggregates. In Fig. 3(b) the polymer and paraffin scattering patterns measured at $273 \mathrm{~K}$ from the second PEB$7.5 / \mathrm{C}_{30}$ solution $\left(\Phi_{\mathrm{pol}}=0.6 \%\right.$ and $\left.\Phi_{\mathrm{wax}}=0.5 \%\right)$ are shown. Characteristic changes are found for the 'polymer' and the 'paraffin' spectra in comparison with the previous case when a higher amount of $\mathrm{C}_{30}$ was investigated and remind us to the scattering of the copolymer in Fig. 1. Both contrast conditions reveal a correlation peak at the same $Q$ as observed for the pure PEB-7.5 solution. This proves that the copolymer dictates to some extent the aggregation behavior of both materials. On the other hand, the aggregation behavior of PEB-7.5 changes from needle-like $\left(\mathrm{d} \Sigma / \mathrm{d} \Omega \simeq Q^{-1}\right)$ to plateletlike $\left(\mathrm{d} \Sigma / \mathrm{d} \Omega \simeq Q^{-2}\right)$ in the presence of paraffin. Thus, the paraffin and copolymer influence each other's aggregation behavior. Similar scattering patterns are depicted in Fig. 4

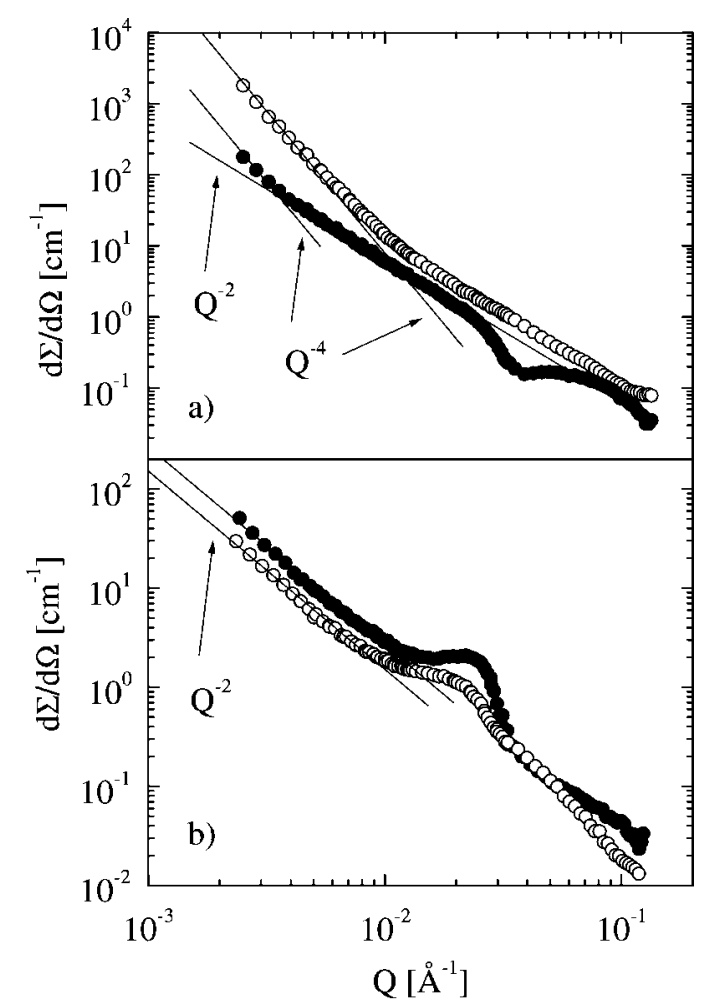

Figure 3

Small-angle scattering cross sections obtained from mixed solutions of PEB-7.5 $\left(\Phi_{\mathrm{pol}}=0.6 \%\right)$ and $\mathrm{C}_{30}$ in d-22 for $(a) \Phi_{\mathrm{wax}}=4 \%$ at $293 \mathrm{~K}$ and $(b)$ $\Phi_{\text {wax }}=0.5 \%$ at $273 \mathrm{~K}$. The solid lines and symbols have the same meaning as in Fig. 2. 
where results from the PEB-7.5/ $\mathrm{C}_{24}$ mixtures are shown for both contrast conditions at $253 \mathrm{~K}$ for the solution with $\Phi_{\text {wax }}=$ $0.5 \%(a)$ and at $273 \mathrm{~K}$ for that with $\Phi_{\text {wax }}=4 \%(b)$. In summary, two different aggregation mechanisms are observed, one of which looks very similar to the pure paraffin-polymer co-crystallization.

Now we will discuss all these results on the basis of a combined phase diagram of paraffins and PEB-7.5 random copolymer in decane. Fig. 5 presents the dissolution lines as a function of the paraffin volume fraction for $\mathrm{C}_{36} \mathrm{H}_{74}, \mathrm{C}_{30} \mathrm{H}_{62}$ and $\mathrm{C}_{24} \mathrm{H}_{50}$ paraffins in decane established by observation of the cloud point (Ashbaugh et al., 2002; Schneiders, 1996). The temperature range where polymer needles are formed is marked as a dashed area, as was concluded from the occurrence of the main features in the polymer self-assembling spectra, namely, the correlation peak and the $Q^{-1}$ power law. The experimental conditions of the SANS results discussed above are also marked with symbols specific to different paraffin solutions. For all solutions (molecule length, temperature, volume fraction) of which the paraffin solubility point is above the polymer self-assembling temperature, a full co-crystallization of the paraffin and PEB-7.5 into twodimensional hairy aggregates occurs. In this case, a higher polymer efficiency in controlling the paraffin crystallization was concluded (Ashbaugh et al., 2002). On the other hand, for the paraffin solutions with their solubility point below the polymer self-assembling temperature, the PEB-7.5

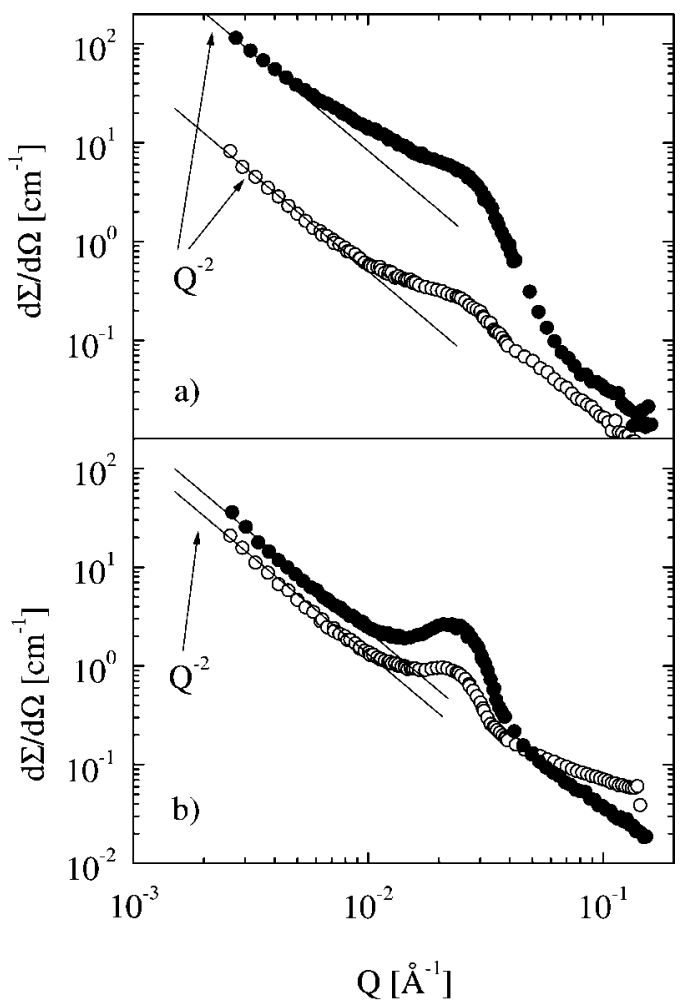

Figure 4

Small-angle scattering cross sections obtained from mixed solutions of PEB-7.5 $\left(\Phi_{\mathrm{pol}}=0.6 \%\right)$ and $\mathrm{C}_{24}$ in $\mathrm{d}-22$ for $(a) \Phi_{\mathrm{wax}}=0.5 \%$ at $253 \mathrm{~K}$ and (b) $\Phi_{\text {wax }}=4 \%$ at $273 \mathrm{~K}$. The solid lines and symbols have the same meaning as in Fig. 2.

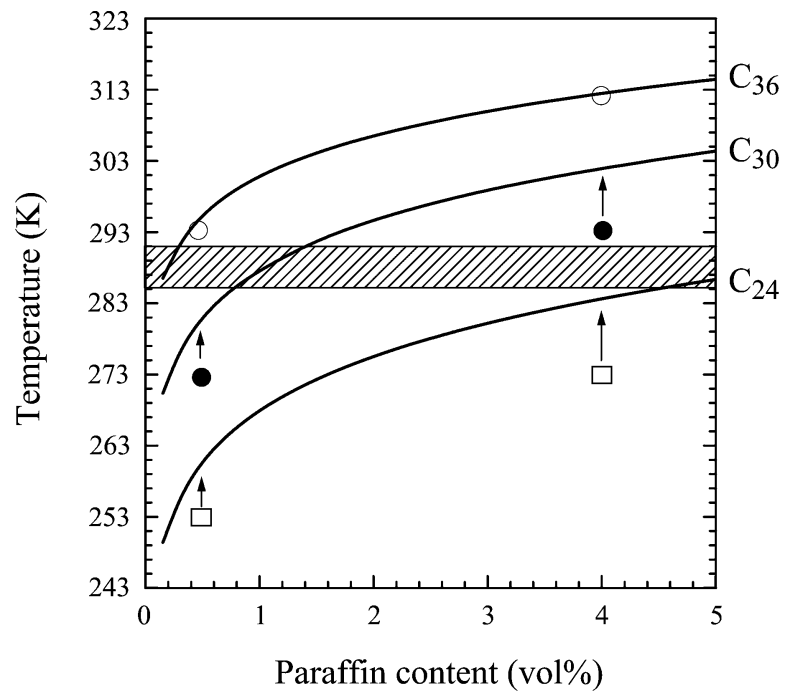

Figure 5

The phase diagram of different $\mathrm{C}_{n}$ paraffins in decane solution showing the paraffin solubility lines. The dashed part indicates the temperature range associated with the appearance of polymer needles. The symbols denote different solution conditions (temperature/paraffin amount) of the SANS investigations discussed within the text for PEB-7.5/ $\mathrm{C}_{36}$ (open circles), PEB-7.5/ $\mathrm{C}_{30}$ (filled circles) and $\mathrm{PEB}-7.5 / \mathrm{C}_{24}$ (open squares) mixtures, respectively.

commences aggregation earlier than the paraffin and forms a primordial structure. This structure is of needle-like shape with density modulations along the axis. The crystalline parts of the polymer needles act as nucleation centers for the paraffin and the polymer coils still in solution, which grow laterally into large two-dimensional correlated objects. Such an arrangement looks very similar to the well known 'shishkebab' morphology found after the crystallization of materials like polyethylene or cellulose from flowing media (Barham, 1993). A lower polymer efficacy in controlling the paraffin crystallization was associated with this mechanism (Ashbaugh et al., 2002).

\section{Summary and conclusions}

The PEB-7.5 random copolymer self-assembles in decane by forming needle-shape objects with a modulated density given by an alternating crystalline-amorphous sequence along the axis. The morphology of the self-assembled structure strongly depends on the precipitation temperatures of the polymer and paraffin molecules. Two aggregation mechanisms have been identified as depending on which component aggregates first. Accordingly, either a co-crystallization of the paraffin and the PEB-7.5 into two-dimensional hairy platelets takes place, or a polymer template structure is formed first, which then acts as the nucleation center for the paraffin molecules.

\section{References}

Ashbaugh, H. S., Radulescu, A., Prud'homme, R. K., Schwahn, D. Richter, D. \& Fetters, L. J. (2002). Macromolecules, 35, 7044-7053. 


\section{research papers}

Barham, P. J. (1993). Crystallization and Morpholgy of Semicrystalline Polymers in Materials Science and Technology, Vol. 12, Structure and Properties of Polymers, edited by E. L. Thomas. Weinheim: $\mathrm{VCH}$.

Higgins, J. S. \& Benoit, H. (1994). Polymers and Neutron Scattering. Oxford: Clarendon Press.

Leube, W., Monkenbusch, M., Schneiders, D., Richter, D., Adamson, D., Fetters, L. J., Dounis, P. \& Legrove, R. (2000). Energy Fuel, 14, 419-430.

Lin, E. K. \& Gast, A. P. (1997). Macromolecules, 29, 4432-4441.

Monkenbusch, M., Schneiders, D., Richter, D., Willner, L., Leube, W., Fetters, L. J., Huang, J. S. \& Min, L. (2000). Physica B, 276-278, 941-943.
Morton, M. \& Fetters, L. J. (1975). J. Rubber Chem. Technol. 48, 359409.

Richter, D., Schneiders, D., Monkenbusch, M., Willner, L., Fetters, L. J., Huang, J. S., Lin, M., Mortensen, K. \& Farago, B. (1997). Macromolecules, 30, 1053-1069.

Schwahn, D., Hahn, K., Streib, J. \& Springer, T. (1990). J. Chem. Phys. 93, 8383-8390.

Schwahn, D., Richter, D., Wright, P. J., Symon, C., Fetters, L. J. \& Lin, M. (2002). Macromolecules, 35, 861-870.

Schwahn, D., Richter, D., Lin, M. \& Fetters, L. J. (2002). Macromolecules, 35, 3762-3768.

Schneiders, D. (1996). PhD thesis, University of Aachen, Germany. 